\title{
The length of susceptibility vessel sign predicts early neurological deterioration in minor acute ischemic stroke with large vessel occlusion
}

\author{
Lanying He ${ }^{1}$, Jian Wang ${ }^{1}$, Feng Wang ${ }^{1}$, Lili Zhang ${ }^{1}$, Lijuan Zhang ${ }^{2}$, Wang Zhao ${ }^{3}$, Xiechuan Weng ${ }^{4^{*}}$ and Fan X u $^{5^{*}}$
}

\begin{abstract}
Background: Patients with acute large vessel occlusion (LVO) presenting with minor stroke are at risk of early neurological deterioration (END). The present study aimed to evaluate the frequency and potential predictors of END in patients with medical management and LVO presenting with minor stroke. The relationship between SVS length and END was also investigated.
\end{abstract}

Methods: This was a prospective multicenter study. Consecutive patients were collected with anterior circulation. LVO presented with minor stroke [National Institutes of Health Stroke Scale (NIHSS) $\leq 4$ ] within $24 \mathrm{~h}$ following onset. END was defined as a deterioration of $\mathrm{NIHSS} \geq 4$ within $24 \mathrm{~h}$, without parenchymal hemorrhage. The length of the susceptibility vessel sign (SVS) was measured using the T2* gradient echo imaging.

Results: A total of 134 consecutive patients with anterior circulation LVO presenting with minor stroke were included. A total of 27 (20.15\%) patients experienced END following admission. Patients with END exhibited longer SVS and higher baseline glucose levels compared with subjects lacking END $(P<0.05)$. ROC curve analysis indicated that the optimal cutoff point SVS length for END was SVS $\geq 9.45 \mathrm{~mm}$. Multivariable analysis indicated that longer SVS [adjusted odds ratio (aOR), 2.03; 95\% confidence interval (Cl), 1.45-2.84; $P<0.001$ ] and higher baseline glucose $(\mathrm{aOR}, 1.02 ; 95 \% \mathrm{Cl}, 1.01-1.03 ; P=0.009)$ levels were associated with increased risk of END. When SVS $\geq 9.45 \mathrm{~mm}$ was used in the multivariate logistic regression, SVS $\geq 9.45 \mathrm{~mm}(\mathrm{aOR}, 5.41 ; 95 \% \mathrm{Cl}, 1.00-29.27 ; P=0.001)$ and higher baseline glucose [aOR1.01; 95\%Cl, 1.00-1.03; $P=0.021$ ] were associated with increased risk of END.

Conclusions: END was frequent in the minor stroke patients with large vessel occlusion, whereas longer SVS and higher baseline glucose were associated with increased risk of END. SVS $\geq 9.45 \mathrm{~mm}$ was a powerful independent predictor of END.

Keywords: Acute minor stroke, Susceptibility vessel sign, Large vessel occlusion, Early neurological deterioration

*Correspondence: wengxc2000@163.com; xufan@cmc.edu.cn

${ }^{4}$ Beijing Institute of Basic Medical Sciences, Beijing 100850, China

${ }^{5}$ School of Public Health Chengdu Medical College, Chengdu 610500,

Sichuan, China

Full list of author information is available at the end of the article

\section{Background}

Patients with minor neurological symptoms of acute ischemic stroke (AIS) are frequent. Neurological symptoms account for approximately half of all patients with AIS $[1,2]$. Although minor strokes are considered benign, nearly one-third of the subjects affected have a persistent disability [3-5]. Previous studies have shown 
that approximately $30 \%$ of minor strokes are caused by acute large vessel occlusion (LVO). The affected patients were at high risk of early neurological deterioration (END) $[6,7]$.

The susceptibility vessel sign (SVS) on gradient echo (GRE) imaging is defined as a hypointense signal exceeding the diameter of the contralateral artery at the site of the thrombus $[8,9]$. The SVS is related to the presence of deoxyhemoglobin, which causes inhomogeneities in local magnetic fields and therefore signal loss on the T2* sequences. Certain studies have suggested that SVS is associated with cardioembolic (CE) subtype [10-12] and the characteristics of SVS, such as their diameter and length, may predict stroke subtypes [13].

Recent studies have shown that SVS is significantly associated with lower early [14] or 24-h $[13,15]$ recanalization rate in the AIS group with intravenous thrombolysis [13-15]. Longer thrombus size was independently associated with END in patients with minor stroke and LVO following intravenous thrombolysis [16].

Accurate prediction of END in patients with minor stroke and large vessel occlusion following medical treatment may aid clinicians to select appropriate treatment, such as thrombectomy.

However, the relationship between SVS length and END in patients with minor AIS and LVO receiving medical treatment has been studied to a lesser extent. Therefore, the current study aimed to evaluate the frequency and potential predictors of END in medically managed patients with LVO presenting with minor AIS. Moreover, the relationship between SVS length and END was investigated.

\section{Methods}

\section{Study population}

The present study was a multicenter prospective study conducted in the three following medical centers: The Second People's Hospital of Chengdu, Yongchuan Hospital of Chongqing Medical University and the Nuclear Industry 416 Hospital. The patients with AIS were admitted within $24 \mathrm{~h}$ of the onset symptoms between May 2014 and September 2020, who were diagnosed as AIS according to the WHO diagnostic criteria and were confirmed with the brain computed tomography $(\mathrm{CT})$ or diffusionweighted imaging (DWI) using Siemens Magnetom Avan to 1.5 Tesla (Siemens Medical Solutions, Erlangen, Germany) on admission. LVO was confirmed with computed tomography angiography (CTA) or magnetic resonance angiography (MRA). The initial severity of stroke was assessed by the National Institutes of Health Stroke Scale (NIHSS).

\section{Inclusion and exclusion criteria}

The patients were enrolled only if they fulfilled the following criteria: 1 . Age $\geq 18$ years; 2 . Admission for firstever acute ischemic stroke within $24 \mathrm{~h}$; 3 . NIHSS $\leq 4$; 4.Evidence for ischemic lesions consistent with clinical presentation; 5. Acute LVO in the anterior circulation (defined by occlusion of the intracranial internal carotid artery, proximal segment of the middle cerebral artery [M1 or M2 trunk], or tandem occlusion). 6. All patients received standard therapy (including antiplatelet agents, statins, blood pressure control, or intravenous thrombolysis). The exclusion criteria were the following: 1. Ongoing infection at admission; 2. A history of previous stroke; 3. Moyamoya; 4. Treatment with endovascular therapy (EVT) prior to END; 5. Cerebral hemorrhage, hypoxia (arterial oxyhemoglobin saturation $<90 \%$ ); 6 . Contraindications for MRI examination.

\section{Clinical assessment and definition of END}

The characteristics and vascular risk factors of the patients were collected. Blood cell counts, lipid and glucose levels were obtained on admission.

END was defined as neurological symptoms with an increase in the total NIHSS $\geq 4$ that was attributed to the underlying LVO (excluding the subjects related to hemorrhagic transformation or medical complications) within $24 \mathrm{~h}$.

MR imaging was performed on a $1.5 \mathrm{~T}$ magnet (Siemens Medical Solutions, Erlangen, Germany) with a phased array head coil. The acquisition parameters for GRE T2* were the following: TR, $800 \mathrm{~ms}$; TE, $30 \mathrm{~ms}$; section thickness, $5 \mathrm{~mm}$; intersection gap, $1 \mathrm{~mm}$; FOV, $250 \times 250 \mathrm{~mm}$; flip angle, $20^{\circ}$. All patients were imaged on the same 1.5-T MRI system and the same parameters for GRE T2 were used in three medical centers.

SVS length was measured and its location was noted according to the predefined arterial segments. The inplane length of the clot corresponded to the distance between the proximal and distal part of the SVS; the length of the clot perpendicular to the axial acquisition plane was obtained by multiplying the number of crosssectional locations where the clot was visible by the slice thickness (Fig. 1).

All generated images were stored on a CD-ROM with the visualization software (Siemens Germany). Two radiologists independently reviewed all MRI images without the clinical data and potential disagreements were resolved by discussion.

\section{Statistical analysis}

Firstly, patients were classified into no END and END groups. The data are presented as median values 

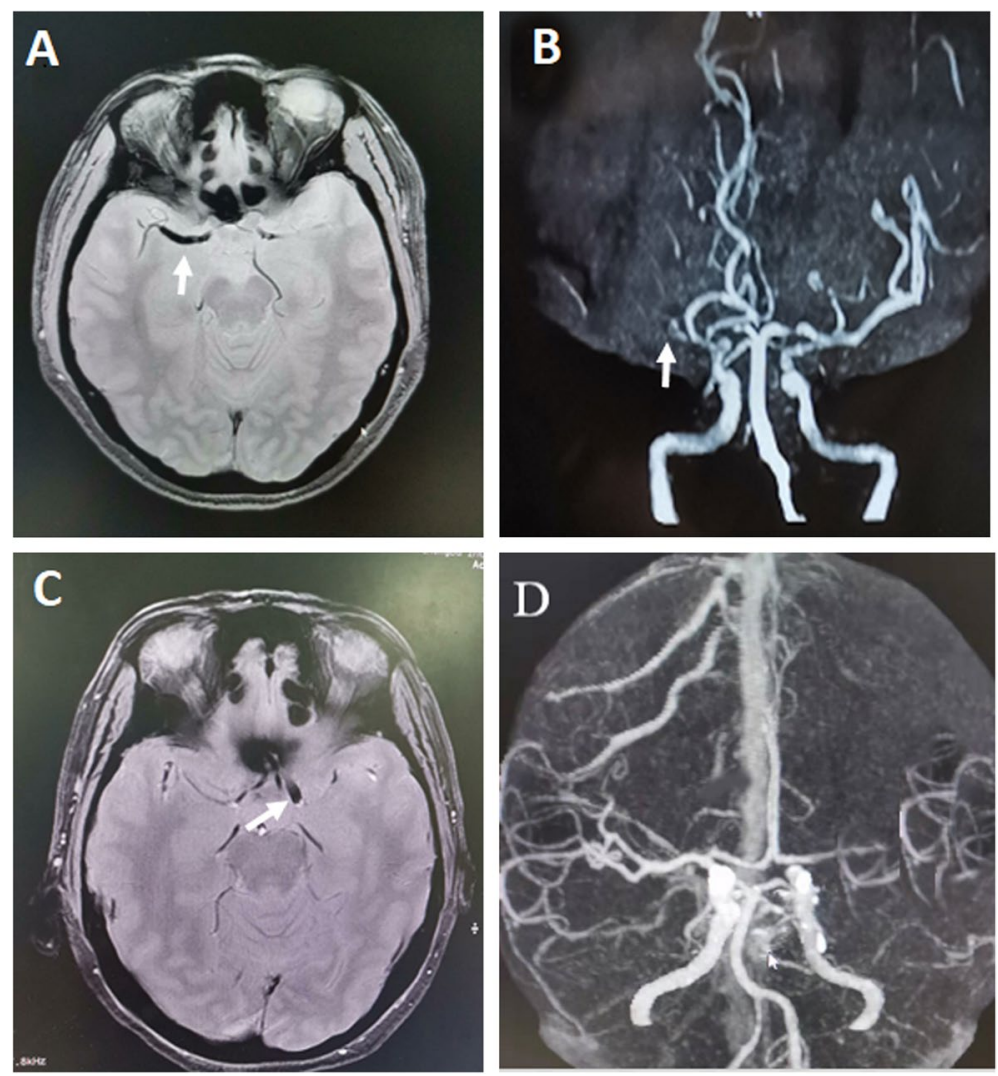

Fig. 1 Examples of long SVS and short SVS. MRI of 2 patients presenting with long SVS (A and B, white arrow) and short SVS (C and D, white arrow); $\mathbf{A}$ and $\mathbf{C}$, axial views of T2*-GRE; $\mathbf{B}$, MRA; D, CTA

(interquartile range $[\mathrm{IQR}])$, numbers (\%), or mean values ( \pm standard deviation). To identify the differences between subgroups, the Pearson $x^{2}$ test was used for categorical variables. The distributions of the continuous variables were determined by the KolmogorovSmirnov test, while the Mann-Whitney two sample test was applied in case of non-normal distributions. Secondly, receiver operating characteristic (ROC) curve analysis was used to evaluate sensitivity, specificity and to determine the optimal cutoff point of length of SVS for END. Thirdly, the variables associated with END in the univariate analyses with a $P$-value $<0.20$ were included in the multivariate analysis. Subsequently logistic regressions analyses were performed to determine the association between specific factors and END. The results were expressed as adjusted odds ratios (aOR) with the corresponding 95\% confidence interval (CI). The data were analyzed using SPSS software (SPASS 22.0). $P<0.05$ was considered to indicate statistically significant differences.

\section{Results}

Characteristics of the study subjects

A total of 174 patients with acute LVO presenting with minor stroke were enrolled at the three centers. Among them, 2 patients demonstrated infection at admission, whereas 17 patients exhibited a history of previous stroke, 1 had Moyamoya disease and 3 had cerebral hemorrhage transformation. A total of 11 subjects had contraindications for MRI examination, while 6 patients were directly transferred for EVT. The remaining 134 patients were recruited. The mean age was $67.01 \pm 10.15$ years (45.8-96.8 years) and the sample size comprised 50\% (67) men. In the study population, 111 patients had a history of hypertension, 51 presented with a history of diabetes, 90 exhibited a history of hyperlipidemia, 24 were current smokers and 33 were current alcohol drinkers. The occlusion sites were intracranial ICA in 34 patients (25.37\%), MCA M1 in 65 patients (48.51\%) and MCA M2 in 35 patients $(26.12 \%)$. The median NIHSS score at admission was 2 (interquartile range, $1-3)$. A total of $35(26.12 \%)$ 
patients underwent intravenous thrombolysis. Among them, $27(20.15 \%)$ patients experienced END following admission.

\section{Evaluation of the prognostic value of SVS for END}

ROC curve analysis indicated high accuracy for SVS in order to predict END with AUC of 0.74 (95\% CI 0.63 to 0.84) (Fig. 2). By using a cutoff value of $S V S \geq 9.45 \mathrm{~mm}$, the sensitivity and specificity was 77.78 and $56.07 \%$, respectively, which provided a positive predictive value (PPV) for END of $30.89 \%$ and a negative predictive value (NPV) of $90.91 \%$.

\section{Univariable models for predictors of END}

A total of 27 (20.15\%) patients experienced END following admission. Patients with SVS $\geq 9.45 \mathrm{~mm}$ experienced more END [30.88\% (21/68) versus 9.09\% (6/66), $P=0.002]$. The baseline characteristics of patients in the no END and END groups were compared (Table 1). At baseline, patients with END indicated significantly higher baseline glucose levels ( $156.88 \pm 43.67$ vs. $128.75 \pm 39.95$, $P<0.001)$, and longer SVS $[10.68(9.70-13.25)$ vs. 9.13(7.73-10.41), $P<0.001]$ than patients with no END. No significant differences were noted in the occlusion site between the groups with or without END $(P>0.05)$.

\section{Multivariable models on the association} between SVS $\geq 9.45 \mathrm{~mm}$ and END

In the unadjusted models, an association between SVS $\geq 9.45 \mathrm{~mm}$ and END was noted (OR, 4.66; 95\%CI, 3.24-6.72, $P<0.001)$. When the factors were associated with END in the univariate analyses $(P<0.20)$ they were used into the multivariate logistic regression analysis (adjustment for SVS value, baseline glucose, hypertension, current smoking, cardiac embolism, undetermined stroke). The results indicated that longer SVS [aOR, 2.03; 95\%CI, 1.45-2.84; $P<0.001]$ and higher baseline glucose (aOR, 1.02; 95\% CI, 1.01-1.03; $P=0.009$ ) were associated with increased risk of END. When SVS $\geq 9.45 \mathrm{~mm}$ was used in the multivariate logistic regression, (aOR, 5.41; 95\%CI, 1.00-29.27; $P=0.001$ ), higher baseline glucose levels (aOR1.01; 95\%CI, 1.00-1.03; $P=0.021$ ] were associated with increased risk of END (Table 2).

\section{Discussion}

The present study indicated that $20.15 \%$ (27) of patients experienced END in minor stroke with LVO. The optimal length of SVS for predicting END was SVS $\geq 9.45 \mathrm{~mm}$. Furthermore, we found that higher baseline glucose levels and longer SVS were associated with increased risk of END in patients with minor stroke and LVO.

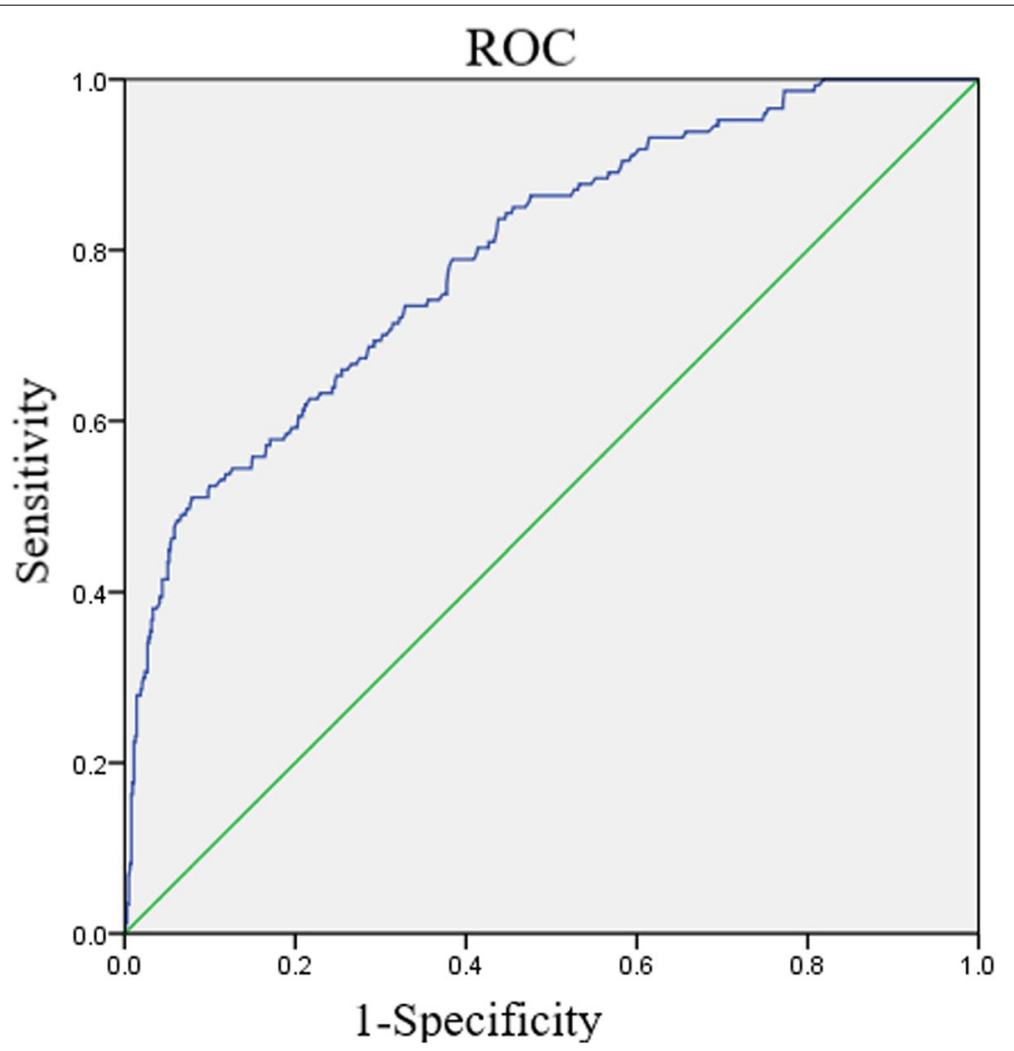

Fig. 2 Receiver operating characteristic curve analysis for SVS. The curve was used for determining the prognostic value of END 
Table 1 Comparison of baseline characteristics between patients with no END and END

\begin{tabular}{|c|c|c|c|c|}
\hline & no END group (107) & END group (27) & $\mathrm{OR}(95 \% \mathrm{Cl})$ & $P^{*}$ \\
\hline Age, y (Mean SD) & $66.75 \pm 10.51$ & $68.01 \pm 8.66$ & & 0.334 \\
\hline NIHSS score, median (IQR) & $2(1-3)$ & $2(1-3)$ & & 0.279 \\
\hline SVS length, median (IQR) & $9.13(7.73-10.41)$ & 10.68(9.70-13.25) & & $<0.001$ \\
\hline $\mathrm{SVS} \geq 9.45 \mathrm{~mm}, \mathrm{n}(\%)$ & $47(43.93)$ & $21(77.78)$ & $4.47(1.67-11.96)$ & 0.002 \\
\hline Time from last seen well to MRI, $\mathrm{h}$ (Mean SD) & $10.5 \pm 7.8$ & $11.2 \pm 7.2$ & & 0.591 \\
\hline Baseline glucose, mg/dl (Mean SD) & $128.75 \pm 39.95$ & $156.88 \pm 43.67$ & & $<0.001$ \\
\hline DWI-ASPECTS, (Mean SD) & $8.98 \pm 1.25$ & $8.93 \pm 1.41$ & & 0.607 \\
\hline Females, n(\%) & $54(50.46)$ & $13(48.15)$ & $0.91(0.39-2.12)$ & 0.829 \\
\hline Men, n(\%) & $53(49.53)$ & 14(51.85) & $0.91(0.39-2.12)$ & 0.829 \\
\hline $\mathrm{BMI} \geq 24 \mathrm{~kg} / \mathrm{m}, \mathrm{n}(\%)$ & $27(25.23)$ & $5(18.52)$ & $0.67(0.23-1.95)$ & 0.465 \\
\hline Hypertension, n(\%) & $91(85.06)$ & $20(74.07)$ & $0.50(0.18-1.38)$ & 0.177 \\
\hline Current Smoking, n(\%) & $22(20.56)$ & $2(7.41)$ & $0.31(0.07-1.41)$ & 0.111 \\
\hline Current alcohol drinking, n(\%) & $27(25.23)$ & $6(22.22)$ & $0.85(0.31-2.32)$ & 0.746 \\
\hline Diabetes, $\mathrm{n}(\%)$ & $42(39.25)$ & $9(33.33)$ & $0.77(0.32-1.88)$ & 0.571 \\
\hline Hyperlipidemia, n(\%) & $73(68.22)$ & 17(62.96) & $0.79(0.33-1.91)$ & 0.603 \\
\hline Thrombolytic therapy, n(\%) & $29(27.10)$ & $6(22.22)$ & $0.77(0.28-2.09)$ & 0.606 \\
\hline Family history of stroke, n(\%) & 14(13.08) & $5(18.52)$ & $1.51(0.49-4.64)$ & 0.469 \\
\hline \multicolumn{5}{|l|}{ Occlusion site } \\
\hline Intracranial ICA, n (\%) & $26(24.30)$ & $8(29.63)$ & $1.31(0.51-3.35)$ & 0.569 \\
\hline MCA M1, n (\%) & $52(48.60)$ & 13(48.18) & $0.98(0.42-2.29)$ & 0.967 \\
\hline MCA M2, n (\%) & $29(27.10)$ & $6(22.22)$ & $0.77(0.28-2.09)$ & 0.606 \\
\hline \multicolumn{5}{|l|}{ TOAST } \\
\hline Cardiac embolism & $22(20.56)$ & $9(33.33)$ & $1.93(0.76-4.88)$ & 0.160 \\
\hline LAA, n (\%) & $30(28.04)$ & $7(25.93)$ & $0.90(0.34-2.34)$ & 0.826 \\
\hline Lacunar, n (\%) & $30(28.04)$ & $8(29.63)$ & $1.08(0.43-2.73)$ & 0.870 \\
\hline Undetermined, n (\%) & $23(21.50)$ & $2(7.40)$ & $0.29(0.064-1.33)$ & 0.093 \\
\hline Other, n (\%) & $2(1.87)$ & $1(3.70)$ & $2.02(0.18-23.13)$ & 0.565 \\
\hline \multicolumn{5}{|l|}{ Medications use } \\
\hline Antiplatelet, n(\%) & $29(27.10)$ & $6(22.22)$ & $0.77(0.28-2.01)$ & 0.606 \\
\hline Antihypertensive, n(\%) & $60(56.07)$ & 14(51.85) & $0.84(0.36-1.97)$ & 0.693 \\
\hline Lipid-lowering medications, n(\%) & $54(50.47)$ & 13(48.15) & $0.91(0.39-2.12)$ & 0.829 \\
\hline
\end{tabular}

Bold indicates $P$-values less than 0.05

*Comparison between no END and END groups. The data are presented as median values (interquartile range [IQR]), numbers (\%), or mean values ( \pm standard deviation). Categorical variables are expressed as frequency (percent) for $P$ values. Continuous variables are expressed as mean \pm standard deviation (SD). Baseline characteristics were compared between the 2 subgroups by univariate analysis using Pearson $x^{2}$ and the distributions of continuous variables were determined by the Kolmogorov-Smirnov test. The Mann-Whitney two sample test was applied in the case of non-normal distributions

Table 2 Multivariable models showing predictors of END

\begin{tabular}{lll}
\hline & aOR $(\mathbf{9 5 \%} \mathrm{Cl})$ & $\boldsymbol{P}^{*}$ \\
\hline $\begin{array}{lll}\text { Model } 1 \text { (SVS length) } \\
\text { SVS length }\end{array}$ & \\
$\quad$ Baseline glucose & $2.03(1.45-2.84)$ & $<\mathbf{0 . 0 0 1}$ \\
Model 2 (SVS $\geq 9.45 \mathrm{~mm})$ & $1.02(1.01-1.03)$ & $\mathbf{0 . 0 0 9}$ \\
SVS $\geq 9.45 \mathrm{~mm}$ & & \\
Baseline glucose & $5.41(1.00-29.27)$ & $\mathbf{0 . 0 0 1}$ \\
\hline
\end{tabular}

Bold indicates $P$-values less than 0.05

* Multivariable adjusted for baseline glucose, hypertension, current smoking, cardiac embolism, undetermined stroke, SVS value or SVS $\geq 9.45 \mathrm{~mm}$
Various acute AIS patients experience neurological deterioration during hospitalization. The common feature for neurological deterioration in these patients is the presence of an underlying LVO [17-20]. According to previous studies, END incidence ranged from 12 to $40 \%$ in patients with minor AIS and LVO [16, 21, 22]. A recent study indicated that the incidence of END in mild stroke patients (NIHSS $\leq 5$ ) with LVO was 19.7\% [22], whereas another study reported that END occurred in $39.4 \%$ in minor stroke and LVO [21]. In a previous study conducted in France, the results indicated that END occurred in $12.1 \%$ patients with minor stroke and LVO 
was intended for intravenous thrombolysis alone [16]. These differences may reflect the clinical environment following stroke, including the inconsistent definitions of neurological deterioration, the medical conditions in the hospital, the differences in nursing conditions and the admission of the patients to the stroke unit. Our findings are in line with the results reported from previously published studies [16, 21-23].

A previous study indicated that the site of occlusion was the predictor of the risk of END, which was reported to be more frequent in the carotid terminus and in the tandem occlusions [23]. However, the applicability of these findings to LVO strokes is questionable since these studies included patients with no LVO stroke, patients with moderate to severe stroke, or exhibited small sample size and inconsistent definitions of END. No statistical differences were noted in the occlusion site between the groups with or without END. These differences may be due to the inclusion of patients with more proximal anterior circulation occlusions (intracranial internal carotid artery, middle cerebral artery M1/2, or tandem occlusion) in the current study. Based on magnetic resonance imaging (MRI) and $\mathrm{T}^{*}$-gradient echo imaging sequence (T2*-gradient echo imaging), the thrombus with high red blood cell content exhibited low signals, which is termed susceptible vessel sign (SVS)+. SVS is related to the presence of deoxyhemoglobin, which leads to the inhomogeneity of local magnetic field and the loss of the T2* signal. Previous studies indicated that decreased SVS length was associated with increased recanalization rate [24-26]. In the present study, we aimed to investigate the association between SVS length and END in patients with minor stroke and LVO. The data demonstrated that longer SVS was associated with increased risk of END in patients with minor stroke and LVO. The END group exhibited a significantly higher SVS than that of the non-END group. The results were consistent with those reported in previous studies [16].

Although the exact mechanisms of END are unclear, one hypothesis is that the symptomatic ischemic tissue (i.e. infarct core and/or penumbra) is extended into the surrounding asymptomatic tissue [27-29]. Only emboli containing deoxyhemoglobin or hemosiderin can be identified as SVS. In other words, SVS is a predictor of older thrombosis. Longer SVS represent a higher clot burden, which is associated with decreased effectiveness of medical treatment and leads to secondary hemodynamic and metabolic disorders. It may be the mechanism of END in patients with minor stroke presented with LVO.

In a previous study, it was shown that thrombus length $>9 \mathrm{~mm}$ was a powerful predictor of lack of early recanalization following intravenous thrombolysis [14].
In the present study, the optimal cutoff point of SVS length for END was SVS $\geq 9.45 \mathrm{~mm}$. However, following adjustment for confounders, a significant association of SVS $\geq 9.45 \mathrm{~mm}$ was noted with risks of END in patients with minor stroke and LVO. Therefore, longer thrombus length (especially $>9 \mathrm{~mm}$ ) was associated with poor prognosis $[14,16]$. The results of the present study indicated that the length of SVS was a valuable marker of END in patients following minor stroke.

It has been shown that patients with hyperglycemia exhibit increased risk for stroke and notably ischemic stroke [30]. Elevated levels of blood glucose in the periinfarct period are closely associated with poor outcome in patients with AIS [31-34]. In patients with END, blood glucose levels were significantly elevated and the possible mechanisms associated with this outcome included poor collateral flow and endothelial cell injury, oxidative stress and lactic acid increase, glucose and energy metabolism disorders. In the present study, higher baseline glucose levels were associated with increased risk of END, which was consistent with previous studies [31-34].

Given the correlation between END and SVS length in patients with mild stroke with LVO, restoration of perfusion is considered a potential treatment strategy for patients at high risk of END. EVT may be a treatment option for patients with long SVS. However, further clinical trials should be designed to demonstrate the benefit of immediate EVT in patients with minor stroke and LVO who are at high risk for END.

The present study contains certain limitations that merit consideration. Firstly, in the present study, data on stroke volume were not available. Secondly, we did not analyze the perfusion images, which may influence the results. Thirdly, SVS was detected using MRI, which required longer time than computed tomography in LVO. MRI was not available for certain stroke patients. However, despite these limitations, the present study was a multicenter clinical study, which required the collection of several important clinical data and the adjustment of data analyses for a wide variety of confounding factors. The results of this study were useful for clinical treatment.

\section{Conclusions}

In conclusion, the present study indicated that END was frequent in minor stroke patients with LVO. Longer SVS and that higher baseline glucose levels were associated with increased risk of END. Moreover, SVS $\geq 9.45 \mathrm{~mm}$ was a powerful independent predictor of END. The END prediction is very important, which can reduce mortality and improve the outcome. Length SVS may have potential predictive value in risk stratification of ischemic stroke, which may help to select 
high-risk patients to initiate intervention in time. The results of the present study imply that this method may be applicable in AIS management.

\author{
Abbreviations \\ Cl: Confidence Interval; M: Mean; OR: Odds Ratio; SD: Standard Deviation; END: \\ Early neurological deterioration; LVO: Large vessel occlusion; NIHSS: National \\ Institutes of Health Stroke Scale; SVS: Susceptibility vessel sign (SVS); IQR: \\ Interquartile range; LAA: Large-artery atherothrombosis; TOAST: Trial of Org \\ 10,172 in Acute Stroke Treatment; EVT: Endovascular therapy; IVT: Intravenous \\ thrombolysis.
}

\section{Acknowledgments}

We thank all patients and their families for generously consenting to the use of human tissues in the current research.

\section{Authors' contributions}

LYH was responsible for the concept and design of the study, data collection and analysis and the first draft of the paper and final manuscript. JW, XW, FX were responsible for the concept and design of the study, data analysis and interpretation. FW was responsible for overseeing the concept and design of the study, data analysis and interpretation and drafting the paper for important intellectual content. LLZ, LJZ and WZ were responsible for data collection. All authors read and approved the final version of the manuscript intended for publication.

\section{Funding}

This work was funded by the Health and Family Planning Commission of Chengdu (2015009), the Chengdu Medical College Natural Science Foundation (CYZ18-33, CYZ19-33), the Sichuan Provincial Education Department (17ZA0134), the Sichuan Medical Association (S18023), the Chengdu Science and Technology Bureau focuses on research and development support plan (2019-YF09-00097-SN), the popular scientific research project of Sichuan Health Commission (20PJ171) and Sichuan undergraduate innovation and startup program funding support (S201913705080, S201913705130, S201913705059, S202013705070, S202013705075, S202013705108). Yunnan Education Bureau (SYSX202036) was not involved in the database management (collection, analysis, interpretation of data) and had no access to patient information. The funding body did not participate in designing the study or writing the manuscript. The study protocol had undergone peer-review process by the funding body.

\section{Availability of data and materials}

The datasets used and/or analyzed during the current study are available from the corresponding author on reasonable request.

\section{Declarations}

\section{Ethics approval and consent to participate}

We obtained ethical approval for this study from the Medical and Health Research Ethics Committee of the Second People's Hospital of Chengdu, Medical and Health Research Ethics Committee of the Yongchuan Hospital of Chongaing Medical University and Medical and Health Research Ethics Committee of Nuclear Industry 416 Hospital. The current study was carried out according to the Declaration of Helsinki. Prior to enrollment, all patients or their legal proxies provided detailed information regarding the aims, scope and possible consequences of the trial by a physician. No diagnostic or interventional procedures were required for the clinical trial. Written informed consent was obtained from all study participants or their legal proxies.

\section{Consent for publication}

Not applicable.

\section{Competing interests}

The authors declare that they have no competing interests.

\section{Author details}

${ }^{1}$ Department of Neurology, The Second People's Hospital of Chengdu, Chengdu 610021, People's Republic of China. ${ }^{2}$ Department of Neurology, The Second Affiliated Hospital of Chengdu College, Nuclear Industry 416 Hospital, Chengdu 610021, People's Republic of China. ${ }^{3}$ Department of Neurology, Yongchuan Hospital, Chongqing Medical University, Chongqing 610020, People's Republic of China. ${ }^{4}$ Beijing Institute of Basic Medical Sciences, Beijing 100850, China. ${ }^{5}$ School of Public Health Chengdu Medical College, Chengdu 610500, Sichuan, China.

\section{Received: 22 April 2021 Accepted: 21 October 2021}

Published online: 29 October 2021

\section{References}

1. Heldner MR, Zubler C, Mattle HP, Schroth G, Weck A, Mono ML, et al. National Institutes of Health stroke scale score and vessel occlusion in 2152 patients with acute ischemic stroke. Stroke. 2013;44(4):1153-7. https://doi.org/10.1161/STROKEAHA.111.000604

2. Reeves M, Khoury J, Alwell K, Moomaw C, Flaherty M, Woo D, et al. Distribution of national institutes of health stroke scale in the Cincinnati/ Northern Kentucky stroke study. Stroke. 2013;44(11):3211-3. https://doi. org/10.1161/STROKEAHA.113.002881.

3. Spokoyny I, Raman R, Ernstrom K, Khatri P, Meyer DM, Hemmen TM, et al. Defining mild stroke: outcomes analysis of treated and untreated mild stroke patients. J Stroke Cerebrovasc Dis. 2015;24(6):1276-81. https://doi. org/10.1016/j.jstrokecerebrovasdis.2015.01.037.

4. Khatri P, Conaway MR, Johnston KC. Acute stroke accurate prediction study (ASAP) investigators. Ninety-day outcome rates of a prospective cohort of consecutive patients with mild ischemic stroke. Stroke. 2012;43(2):560-2. https://doi.org/10.1161/STROKEAHA.110.593897.

5. Romano JG, Smith EE, Liang L, Gardener H, Camp S, Shuey L, et al. Outcomes in mild acute ischemic stroke treated with intravenous thrombolysis: a retrospective analysis of the get with the guidelines-stroke registry. JAMA Neurol. 2015;72(4):423-31. https://doi.org/10.1001/jamaneurol. 2014.4354.

6. Rajajee V, Kidwell C, Starkman S, Ovbiagele B, Alger JR, Villablanca P, et al. Early MRI and outcomes of untreated patients with mild or improving ischemic stroke. Neurology. 2006;67(6):980-4. https://doi.org/10.1212/01. wnl.0000237520.88777.71.

7. Kim JT, Park MS, Chang J, Lee JS, Choi KH, Cho KH. Proximal arterial occlusion in acute ischemic stroke with low NIHSS scores should not be considered as mild stroke. PLoS One. 2013;8(8):e70996. https://doi.org/10. 1371/journal.pone.0070996.

8. Brinjikji W, Duffy S, Burrows A, Hacke W, Liebeskind D, Majoie CBLM, et al. Correlation of imaging and histopathology of thrombi in acute ischemic stroke with etiology and outcome: a systematic review. J Neurointerv Surg. 2017;9(6):529-34. https://doi.org/10.1136/neurintsurg-2016-012391.

9. Liebeskind DS, Sanossian N, Yong WH, Starkman S, Tsang MP, Moya AL, et al. CT and MRI early vessel signs reflect clot composition in acute stroke. Stroke. 2011;42(5):1237-43. https://doi.org/10.1161/STROKEAHA. 110.605576.

10. Horie N, Tateishi Y, Morikawa M, Morofuji Y, Hayashi K, Izumo T, et al. Acute stroke with major intracranial vessel occlusion:characteristics of cardioembolism and atherosclerosis-related in situ stenosis/occlusion. J Clin Neurosci. 2016;32:24-9. https://doi.org/10.1016/j.jocn.2015.12.043.

11. Kim SK, Yoon W, Kim TS, Kim HS, Heo TW, Park MS. Histologic analysis of retrieved clots in acute ischemic stroke: correlation with stroke etiology and gradient-echo MRI. AJNR Am J Neuroradiol. 2015;36(9):1756-62. https://doi.org/10.3174/ajnr.A4402.

12. Liebeskind DS, Sanossian N, Yong WH, Starkman S, Tsang MP, Moya AL, et al. Saver JL.CT and MRI early vessel signs reflect clot composition in acute stroke. Stroke. 2011;42(5):1237-43. https://doi.org/10.1161/STROK EAHA.110.605576.

13. Liu M, Li L, Li G. The different clinical value of susceptibility vessel sign in acute ischemic stroke patients under different interventional therapy: a systematic review and meta-analysis. J Clin Neurosci. 2019;62:72-9. https://doi.org/10.1016/j.jocn.2019.01.002.

14. Seners P, Delepierre J, Turc G, Henon H, Piotin M, Arquizan C, et al. Thrombus length predicts lack of post-thrombolysis early recanalization 
in minor stroke with large vessel occlusion. Stroke. 2019;50(3):761-4. https://doi.org/10.1161/STROKEAHA.118.023455.

15. Legrand L, Naggara O, Turc G, Mellerio C, Roca P, Calvet D, et al. Clot burden score on admission T2*-MRI predicts recanalization in acute stroke. Stroke. 2013;44(7):1878-84. https://doi.org/10.1161/STROKEAHA.113. 001026.

16. Seners P, Ben Hassen W, Lapergue B, Arquizan C, Heldner MR, Henon H, et al. Prediction of early neurological deterioration in individuals with minor stroke and large vessel occlusion intended for intravenous thrombolysis alone. JAMA Neurol. 2021;78(3):321-8. https://doi.org/10.1001/ jamaneurol.2020.4557.

17. Rajajee V, Kidwell C, Starkman S, Ovbiagele B, Alger JR, Villablanca P, et al. Early MRI and outcomes of untreated patients with mild or improving ischemic stroke. Neurology. 2006;67(2):980-4. https://doi.org/10.1212/01. wnl.0000237520.88777.71.

18. Kim JT, Park MS, Chang J, Lee JS, Choi KH, Cho KH. Proximal arterial occlusion in acute ischemic stroke with low NIHSS scores should not be considered as mild stroke. PLoS One. 2013;8:e70996. https://doi.org/10. 1371/journal.pone.0070996.

19. Kim JT, Heo SH, Yoon W, Choi KH, Park MS, Saver JL, et al. Clinical outcomes of patients with acute minor stroke receiving rescue IA therapy following early neurological deterioration. J Neurointerv Surg. 2016;8(5):461-5. https://doi.org/10.1136/neurintsurg-2015-011690.

20. Griessenauer CJ, Medin C, Maingard J, Chandra RV, Ng W, Brooks DM, et al. Endovascular mechanical thrombectomy in large-vessel occlusion ischemic stroke presenting with low national institutes of health stroke scale: systematic review and meta-analysis. World Neurosurg. 2018;110:263-9. https://doi.org/10.1016/j.wneu.2017.11.076.

21. Gwak DS, Kwon JA, Shim DH, Kim YW, Hwang YH. Perfusion and diffusion variables predict early neurological deterioration in minor stroke and large vessel occlusion. J Stroke. 2021;23(1):61-8. https://doi.org/10.5853/ jos.2020.01466.

22. Saleem Y, Nogueira RG, Rodriques GM, Kim S, Sharashidze V, Frankel M, et al. Acute neurological deterioration in large vessel occlusions and mild symptoms managed medically. Stroke. 2020;51(5):1428-34. https://doi. org/10.1161/STROKEAHA.119.027011.

23. Mazya MV, Cooray C, Lees KR, Toni D, Ford GA, Bar M, et al. Minor stroke due to large artery occlusion. When is intravenous thrombolysis not enough? Results from the SITS International Stroke Thrombolysis Register. Eur Stroke J. 2018;3(1):29-38. https://doi.org/10.1177/2396987317746003.

24. Kimura K, Iguchi Y, Shibazaki K, Watanabe M, Iwanaga T, Aoki J. M1 susceptibility vessel sign on $\mathrm{T}^{*}$ as a strong predictor for no early recanalization after IV-t-PA in acute ischemic stroke. Stroke. 2009;40(9):3130-22. https://doi.org/10.1161/STROKEAHA.109.552588.

25. Aoki J, Kimura K, Shibazaki K, Sakamoto Y, Saji N, Uemura J. Location of the susceptibility vessel sign on T2*-weighted MRI and early recanalization within 1 hour after tissue plasminogen activator administration. Cerebrovasc Dis Extra. 2013;3(1):111-20. https://doi.org/10.1159/ 000354848

26. Cho KH, Kim JS, K won SU, Cho AH, Kang DW. Significance of susceptibility vessel sign on $\mathrm{T}^{*}$-weighted gradient echo imaging for identification of stroke subtypes. Stroke. 2005;36(1):2379-83. https://doi.org/10.1161/ 01.STR.0000185932.73486.7a.

27. Alawneh JA, Moustafa RR, Baron JC. Hemodynamic factors and perfusion abnormalities in early neurological deterioration. Stroke. 2009;40(6):e44350. https://doi.org/10.1161/STROKEAHA. 108.532465.

28. Tisserand M, Seners P, Turc G, Legrand L, Labeyrie MA, Charron S, et al. Mechanisms of unexplained neurological deterioration after intravenous thrombolysis. Stroke. 2014;45(12):3527-34. https://doi.org/10.1161/ STROKEAHA.114.006745.

29. Fu J, Zhou Y, Li Q, Zhong G, Zhang S, Zhang R, et al. Perfusion changes of unexplained early neurological deterioration after reperfusion therapy. Transl Stroke Res. 2020;11(2):195-203. https://doi.org/10.1007/ s12975-019-00723-W.

30. Wang F, Jiang B, Kanesan L, Zhao Y, Yan B. Higher admission fasting plasma glucose levels are associated with a poorer short-term neurologic outcome in acute ischemic stroke patients with good collateral circulation. Acta Diabetol. 2018;55(7):703-14. https://doi.org/10.1007/ s00592-018-1139-6.

31. Gordon WR, Salamo RM, Behera A, Chibnall J, Alshekhlee A, Callison RC, et al. Association of blood glucose and clinical outcome after mechanical thrombectomy for acute ischemic stroke. Interv Neurol. 2018;7(3-4):1828. https://doi.org/10.1159/000486456.

32. Simonsen CZ, Schmitz ML, Madsen MH, Mikkelsen IK, Chandra RV, LeslieMazwi T, et al. Early neurological deterioration after thrombolysis: clinical and imaging predictors. Int J Stroke. 2016;11(7):776-82. https://doi.org/ $10.1177 / 1747493016650454$

33. Huang ZX, Huang Y, Zeng J, Hao H, Petroski GF, Lu H, et al. Admission glucose levels may increase the risk for early neurological deterioration in females with acute ischemic stroke. Front Neurol. 2020;11:548892. https:// doi.org/10.3389/fneur.2020.548892.

34. Yi X, Wang C, Liu P, Fu C, Lin J, Chen Y. Antiplatelet drug resistance is associated with early neurological deterioration in acute minor ischemic stroke in the Chinese population. J Neurol. 2016;263(8):1612-9. https:// doi.org/10.1007/s00415-016-8181-5.

\section{Publisher's Note}

Springer Nature remains neutral with regard to jurisdictional claims in published maps and institutional affiliations.
Ready to submit your research? Choose BMC and benefit from:

- fast, convenient online submission

- thorough peer review by experienced researchers in your field

- rapid publication on acceptance

- support for research data, including large and complex data types

- gold Open Access which fosters wider collaboration and increased citations

- maximum visibility for your research: over $100 \mathrm{M}$ website views per year

At BMC, research is always in progress.

Learn more biomedcentral.com/submissions 